\title{
System for monitoring UV radiation level in phototherapy cabins in Poland
}

Joanna Narbutt' ${ }^{1}$, Mariola Pawlaczyk², Anna Sysa-Jędrzejowska³, Janusz W. Krzyścin", Piotr Sobolewski ${ }^{4}$, Bonawentura Rajewska-Więch ${ }^{4}$, Aleksandra Lesiak ${ }^{1}$

${ }^{1}$ Department of Dermatology, Medical University of Lodz, Lodz, Poland ${ }^{2}$ Department of Biology and Environmental Study, Poznan University of Medical Sciences, Poznan, Poland

${ }^{3}$ University of Social Sciences, Lodz, Poland

${ }^{4}$ Institute of Geophysics, Polish Academy of Sciences, Warsaw, Poland

Submitted: 27 June 2012

Accepted: 10 October 2012

Arch Med Sci 2014; 10, 6: 1244-1254

DOI: 10.5114 /aoms.2014.47834

Copyright () 2014 Termedia \& Banach

\section{Abstract}

Introduction: Ultraviolet phototherapy (UVP) is widely used in dermatological practice for the treatment of various skin diseases. Numerous studies support its beneficial curing effectiveness; however, overexposure to ultraviolet radiation can cause adverse health effects, such as sunburn reaction, erythema response, cataract, skin aging, etc. For these reasons, it is of special importance to monitor performance of UVP cabins using a calibration system to evaluate the UV doses incident upon the patient.

Material and methods: A mechanized cabin control system (CCS) is proposed. It consists of radiometers with a wide and narrow field of view to estimate the body irradiation and to identify malfunctioning cabin tubes. Quality control and quality assurance procedures are developed to keep high accuracy of the calibration procedure. The CCS has been used in the examination of two different types of UVP cabins routinely working in Poland.

Results: It allows precise calculation of UV doses and spatial variability of UV radiance inside the cabin, thus providing uncertainties of the doses assigned by medical staff. The CCS could potentially serve as a primary standard for monitoring various UVP cabins working in Poland.

Conclusions: The methodology developed to quantify UV doses in UVP cabins may be easily extended to any UV radiation source.

Key words: phototherapy, UV radiation, medical cabinet, dose calibration.

\section{Introduction}

Ultraviolet (UV) therapy has been used for decades in the treatment of skin diseases, mainly psoriasis, vitiligo, cutaneous T-cell lymphoma, and eczema [1, 2]. The effectiveness of artificial UV light in different skin therapies is well documented [3, 4]. UV therapy cabins are used in dermatology worldwide. Various fluorescent emitting tubes, e.g. narrowband UVB (311 nm) - TL01, broadband UVB - TL12, and broadband UVA - TL09 (for psoralen UVA therapy), could be arranged in a round assembly to provide strong UV radiation exceeding the maximum of the solar irradiance that reaches the Earth's surface.

Determination of the irradiance at the patient's skin surface requires the use of calibrated radiometers [5]. The radiometers contain filters to select the waveband corresponding to that of the cabin UV tubes and have

\author{
Corresponding author: \\ Janusz W. Krzyścin \\ Institute of Geophysics \\ Polish Academy of Sciences \\ 64 Księcia Janusza St \\ 01-452 Warsaw, Poland \\ Phone: +48 226915874 \\ Fax +48 226915915 \\ E-mail: jkrzys@igf.edu.pl
}


specific spectral sensitivity varying significantly between sensors made even by the same manufacturing company. Thus, a comparison of the radiometer reading with irradiance measurements from a calibrated spectroradiometer and measurements of the radiometer spectral sensitivity are necessary to convert output of the radiometer (in volts) to the irradiance scale $\left(\mathrm{mW} \cdot \mathrm{cm}^{-2}\right)$ [5]. Martin et al. [6] reported variations of $50 \%$ in the cabinet irradiance measurements made by different radiometers having the same calibration procedure with a high resolution spectrophotometer. The differences were caused by specific angular properties of the radiometer detector, and radiometers with good angular responses should have been recommended for the cabinet measurements [7]. Curie et al. [8] proposed an automated dosimetry system for testing whole-body ultraviolet phototherapy in dermatological cabins and demonstrated variations in the irradiance measured inside the cabin. The system has not been put into operational use probably because of its calibration procedure that requires regular comparisons of the system UV detectors with a high resolution spectroradiometer during simultaneous measurements in a dermatological cabin.

Accurate estimation of the UV dose received by a patient during phototherapy remains a problem. One possible method is to measure the cabin UV intensity at different body points performed by a UV-protected operator using a personal handheld dosimeter calibrated for the spectrum of the tubes used [9]. The measured doses are then averaged and this mean value represents the cabin irradiance, which is then multiplied by a patient's exposure duration to estimate the UV radiation dose received during the phototherapy treatment. This method is characterized by a large uncertainty and the calibrations differ markedly between specialist centers. For example, TL-01 calibration in the UK varies by at least a factor of 2.7 [10]. There were clinics, however, which either did not have reliable hand-held dosimeters or did not trust their output, and in order to obtain an estimation of patient dosage they performed measurements using a radiometer built in a cabin and computer software evaluating the dosage from this radiometer data or delivered the patient a dosage based on exposure time only [4]. Such methodology was also routinely implemented in two examined cabins in Poland, one located in Poznan (later in the text referred to as Poznan phototherapy cabin, PPC) and another in Lodz (LPC). The dose received by a patient was based on output of the built-in dosimeter in PPC, while in LPC it was based on the exposure time. It is of special importance to control and quantify the patient's radiation dose to ensure both the safety and the effectiveness of the cabin therapy.
In this paper we propose a cabin calibration system (CCS) to improve the current dosimetry methods used in Poland and to aid the patient treatments in order to maximize therapeutic efficiency. The proposed system is based on the Curie et al. [8] calibration concept of using two UV sensitive instruments rotating inside the cabinet to determine the doses received by a patient during the medical treatment and to identify malfunctioning tubes. In our solution, different instruments of the system are used and we propose a novel calibration procedure of radiometers measuring the UV irradiance by fluorescent tubes. Details of the UV distribution in the cabin and the tube aging process are also discussed with a view to extending our knowledge of the long-term performance of the cabin.

\section{Material and methods}

\section{The phototherapy cabins}

Two types of medical cabins were examined: MEDISUN 2800 PC-AB in Lodz, and MEDISUN2800-44-PC-AB in Poznan. The whole body phototherapy was performed in those cabins using narrow band UVB (TL-01) and broadband UVA (TL-09 UV-A) fluorescent tubes (Philips Lighting B.V., Roosendall, the Netherlands). The irradiance spectra of the tubes, measured inside the cabin by a USB2000+ spectrophotometer (Ocean Optics Inc., Dunedin, Florida, USA), are shown in Figure 1. There are 12 TL-01 and 16 TL-09, and 16 TL-01 and 32 TL-09 tubes mounted in a round assembly in LPC and PPC, respectively, as presented in Figure 2.

In Lodz the UV dose $\left(J \cdot \mathrm{cm}^{-2}\right)$ received by a patient during the phototherapy treatment is estimated using a joulemeter. The duration of the cabin irradiation of a patient compliant with the UV dose prescribed by a medical doctor is set by nursing staff. Thus, a constant intensity of the tube radiation is assumed and the received UV dose is proportional to the duration of the cabin treatment.

The duration of the irradiation in the Poznan cabin is determined according to the prescribed individual patient dose and the measured irradiation by the built-in cabin radiometer. In such a way a loss of the tube power due to the aging processes, which may happen after prolonged use of the tubes, may be compensated by a longer duration of the phototherapy.

\section{Cabin control system}

We examined the performance of the tubes using the CCS, which consists of a commercial meter, YES UVA-1 ultraviolet pyranometers (Yankee Environmental System Inc., Turner Fall, MA, USA), 

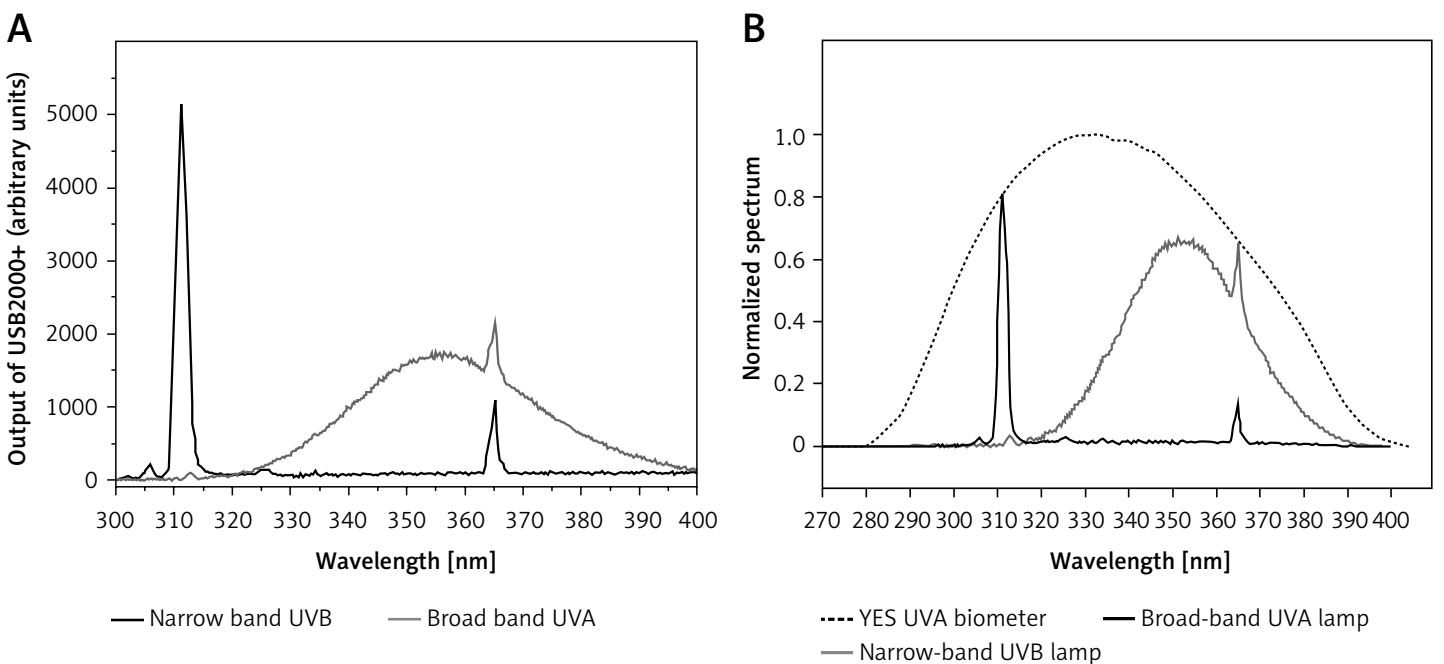

Figure 1. Spectra of the radiation by the narrow band UVB TL-01 and broadband UVA TL-09 tubes used in the phototherapy cabin in Lodz and Poznan (A). The normalized sensitivity spectrum of YES UVA-1 meter and the tube spectra weighted by the normalized sensitivity of YES UVA-1 meter (B)
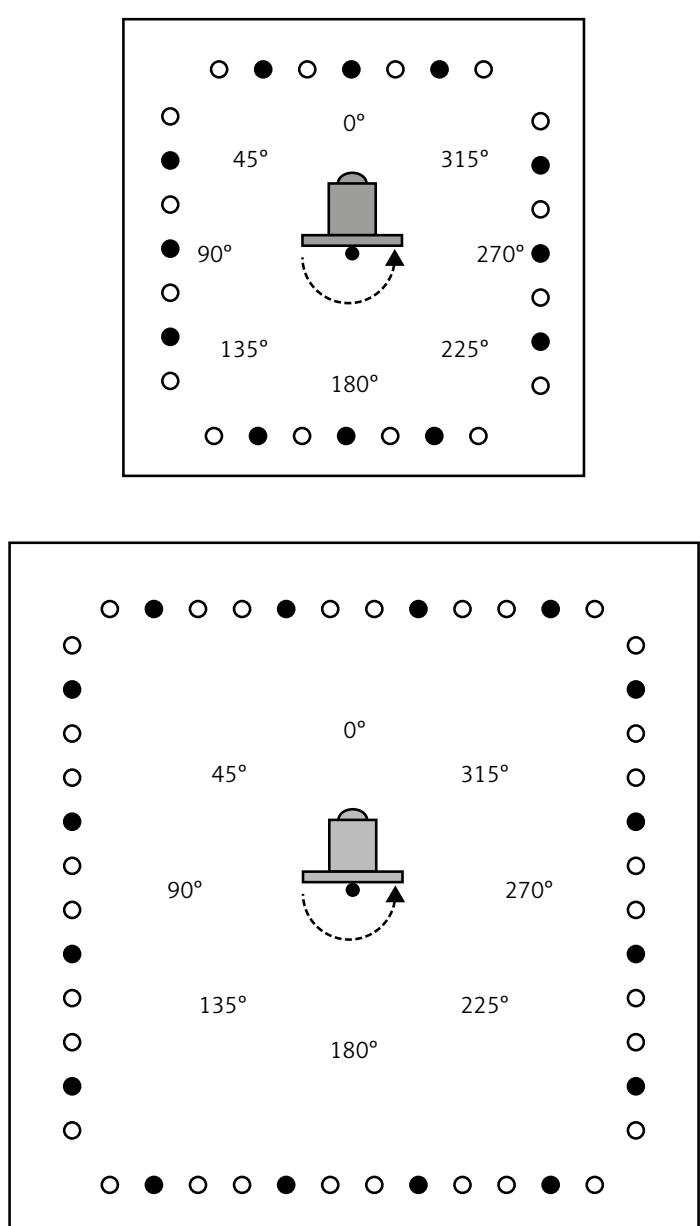

Figure 2. Tube configuration in MEDISUN-2800 PC-AB (Lodz, top) and MEDISUN-2800-44-PC AB (Poznan, bottom) cabin and position of the calibration unit in the centre. Full and open circles mark narrow band UVB TL-01 tubes and broad band UVA TL-09 tubes, respectively. The arrows show the anticlockwise rotation of the calibration unit. The rotation angles are shown round in the circle with a wide field of view (almost hemispherical) to estimate the irradiation (in $\mathrm{mW} \cdot \mathrm{cm}^{-2}$ ) on the surface, and two prototype meters, developed by the Tele \& Radio Research Institute, Warsaw, containing Schottky diodes with a narrow viewing angle $\left(\sim 40^{\circ}\right)$ to identify small-scale spatial variability in UV radiation field in the cabin. One of the narrow viewing angle (NVA) meters contains a GaN/ AIGaN diode which is sensitive to UVB radiation from TL-01 tubes. The other one, with a GaN/ AIGaN p-i-n diode that measures mostly UVA radiation, was designed to monitor TL-09 tubes. Below we use the acronyms NVA-UVB and NVA-UVA, respectively, to denote the two meters.

The UV monitoring instruments were mounted on a remote control rotating device, which allows one to quantify UV irradiance coming from different sectors of the cabin. The automated system provides a reproducible technique for cabin monitoring and reveals more detailed information on the UV irradiance distribution within the cabin. The details of the CCS calibration procedure are included in the supplementary information section for this article.

\section{UV measurements in the cabin}

The measurements of the UV irradiation in the center of the cabin were carried out by the CCS mounted on a vertical bar (Figure 3) that could rotate due to the motorized system. The CCS measured the UV changes inside the cabin during $360^{\circ}$ rotation at 3 selected vertical levels corresponding to the height of a human face, chest, and stomach. The measurements of the UV intensity by the CCS consisted of the following stages:

1) fixed position (in front of one of the cabin walls) for about $60 \mathrm{~s}$, 
2) rotation in the anticlockwise direction to make a full $\left(360^{\circ}\right)$ circle for about $240 \mathrm{~s}$,

3 ) fixed position as in stage 1 ; the resting time lasts about $60 \mathrm{~s}$,

4) rotation in the clockwise direction and after about $240 \mathrm{~s}$ arrival at the initial position (stage 1 ),

5) fixed position for about $60 \mathrm{~s}$ as in stage 1.

The procedure, referred to below as Mode $A$ of the cabin examination, was used to estimate the UV variability inside the cabin. The measuring sensor was placed at the distance of $15 \mathrm{~cm}$ from the rotating axe (Figure 3 ), as the irradiated parts of the patient's body are usually outside the geometric centre of the cabin. The effects of the patient's presence in the cabin on the UV level were examined using a manikin. The manikin absorbs part of the radiation and reduces the multiple reflectance between the cabin walls. There are two options: one with an undressed and another with a dressed manikin (Figure 4). During the experiment, the CCS was fixed as in stage 1 , and the manikin stood just behind the measuring unit. The fixed position of the CCS in front of the cabin wall is referred to as Mode $B$ of the cabin examination.

The CCS is designed to compare the UV doses with nominal doses previously assigned by nursing staff. The selected nominal doses were $1.4 \mathrm{~J}$ - $\mathrm{cm}^{-2}$ and $5 \mathrm{~J} \cdot \mathrm{cm}^{-2}$ for TL-01 and TL-09 tubes in $\mathrm{LPC}$, respectively. In PPC only TL-01 tubes were examined, and a $2.0 \mathrm{~J} \cdot \mathrm{cm}^{-2}$ dose was selected for the calibrations because the broad band UVA phototherapy by TL-09 tubes is rarely prescribed to a patient. The selected nominal doses correspond to those used in medical practice $[11,12]$.
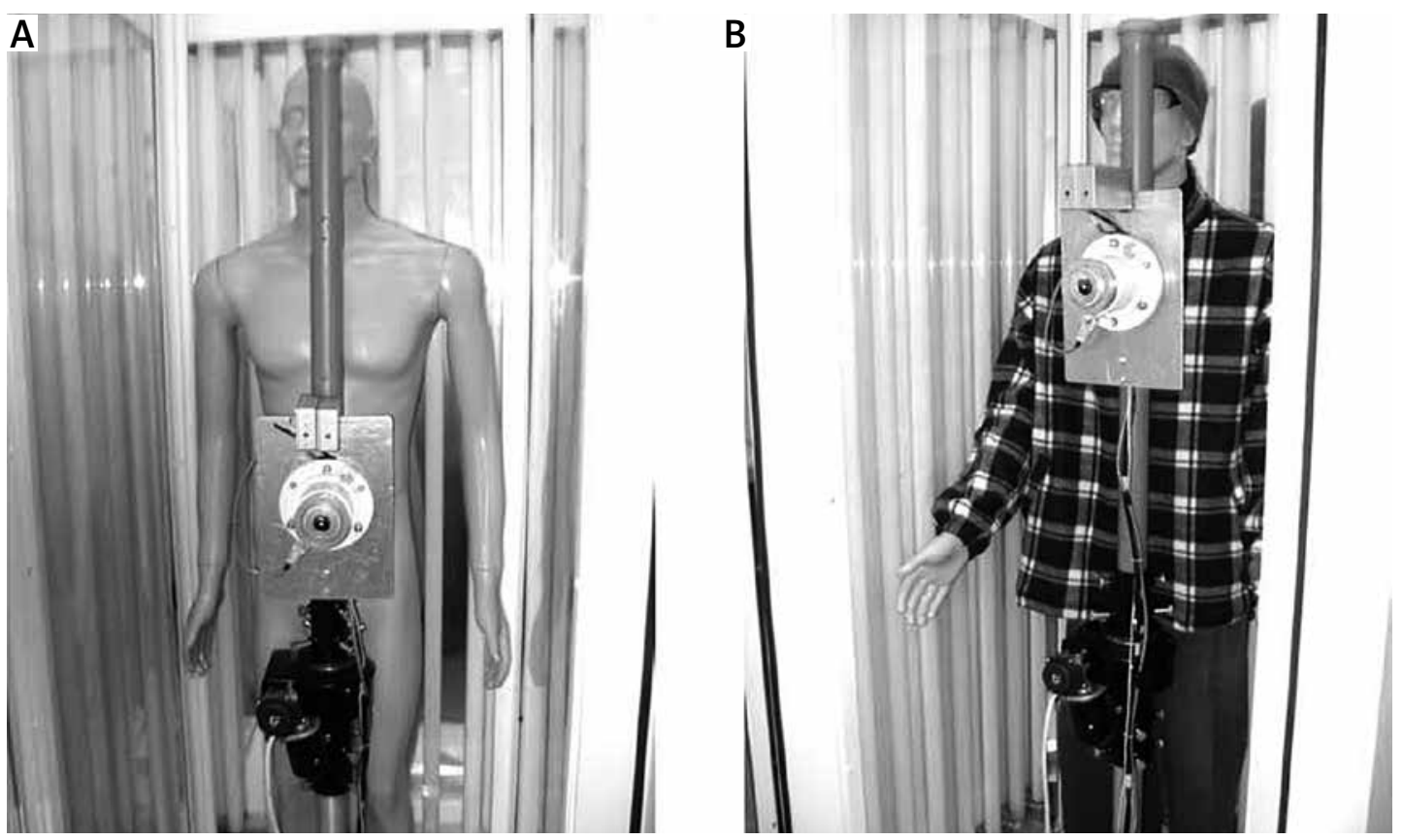

Figure 3. The calibration meters mounted on a vertical plate that is attached to a rotating vertical beam. Its rotation is due to the motorized system (bottom)

\section{Results}

\section{Cabinet measurements}

Examples of the UV irradiance emitted by TL-01 tubes in the experiments in Lodz and Poznan performed in Mode A are shown in Figures 5. Figure 5 A shows the irradiance time series by YES UVA-1

Figure 4. The calibration session in the phototherapy cabin in Lodz with naked (A) and dressed manikin (B) 
measured at each stage of Mode A. In Lodz, in stage 1 ( $1^{\text {st }}$ min of the cabinet operation), the UV irradiance increased only slightly during warming of the tubes. In Poznan, the measurements in PPC started with a jump after which the cabin software adjusts the UV intensity to lower values, and a few seconds later a slight upward tendency in the tube irradiation appears. The irradiance fluctuated in stages 2 and 4 because the radiometer measured the UV radiation coming from different sectors of the cabin walls. The UV level in the Poznan cabin was higher because PPC contains more tubes, 16, in comparison with LPC, 12. The range of UV fluctuations, which was meaand the minimum irradiance recorded during the CCS rotations, was $\sim 1 \mathrm{~mW} \cdot \mathrm{cm}^{-2}$ in LPC, being twice as large as that in PPC. In stages 3 and 5 the irradiance level was almost constant. The calibration procedure (Mode A) of the cabin status lasted about $11 \mathrm{~min}$. The irradiance level at the end of the procedure was only slightly lower than that at the start.

Figure 5 B shows the irradiance variability in stages 2 and 4, i.e., only during the CCS rotation, measured by the NVA-UVB meter in LPC and PPC. In this case the UV radiance incident upon the instrument sensor comes from a small area of the cabin, as the viewing angle of the instrument is about $40^{\circ}$. The variability of UV radiation was much higher compared to that obtained by YES UVA-1. In certain positions (cabinet corners), the NVA-UVB meter registered only UV radiation reflected from the cabinet walls outside the influence of direct lamp radiation. The irradiance field in the cabin was smoother in PPC because more sured as the difference between the maximum

tubes were used. During the clockwise rotation of the CCS in LPC (step 4 of Mode A) the irradiance was only slightly lower than that during anticlockwise rotation (step 2 of Mode A). This was probably due to lower efficiency of the tube at higher temperature and/or due to the temperature sensitivity of semiconductors used in NVA meters. The computer software controls the tube efficiency in PPC taking into account irradiation measured by the built-in UV meter. Thus, sometimes the irradiance during the clockwise turning is slightly higher than that during the anticlockwise turning.

The examination of the cabin with NVA meters allowed the performance of individual tubes to be monitored and indicated a loss of their power due to aging of the tubes. Thus, it seems also possible to identify the malfunctioning tubes. YES UVA-1 is more appropriate to determine the irradiation of a patient body, as it is sensitive to radiance coming from a wide hemispherical area of the cabin that corresponds to the field-of-view of the patient's skin during the phototherapy.

\section{Measured versus nominal doses in the empty cabin}

Table I shows the ratio between nominal doses that a patient should receive during phototherapy in LPC and the actual dose measured at different levels corresponding to the height of the stomach, chest, and face of a patient $175 \mathrm{~cm}$ tall. The results are for two data sets extracted from Mode A measurements:

- full series of measurements comprising all stages (1-5) of Mode A,

B

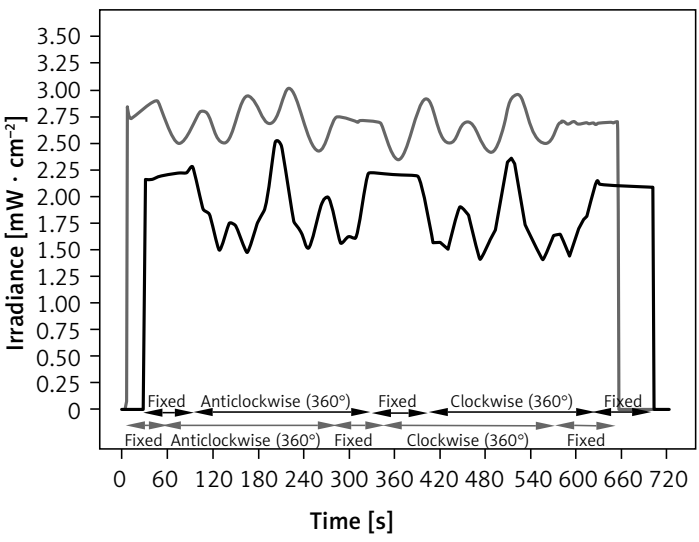

- Poznan, 9.04.2010

— Lodz, 19.06.2010

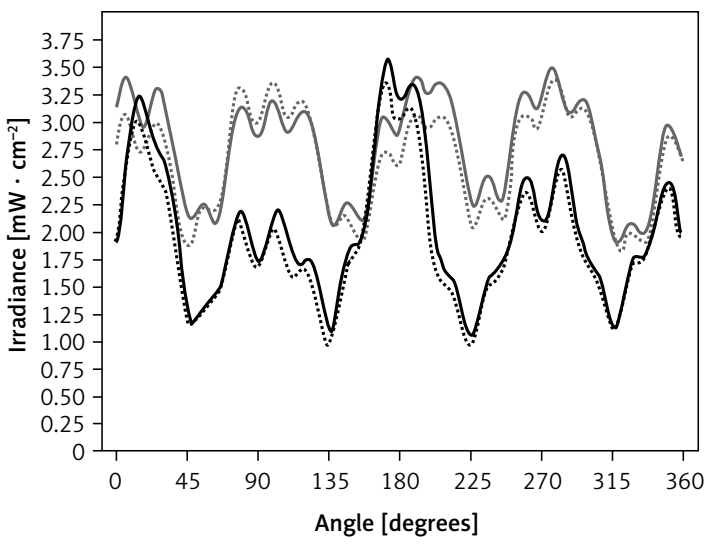

— Poznan, anticlockwise, 9.04.2010

-.-. Poznan, clockwise, 9.04.2010

— Lodz, anticlockwise, 19.06.2010

---. Lodz, clockwise, 19.06.2010

Figure 5. The irradiance time series measured during the calibration of narrow band tubes in phototherapy cabins in Lodz and Poznan: Mode A of the calibration schedule by YES UVA-1 radiometer (A), step 2 and 4 of Mode A by the narrow viewing angle instrument NVA UVB (B) 
Table I. Ratio between nominal and measured doses in Lodz cabin (MEDISON 2800-PC AB) obtained during 3 comparison campaigns in 2010 for the narrow band UVB TL-01 tubes (NUVB) and broadband UVA TL-09 tubes (BUVA). The total work time since the last replacement of all tubes is shown in the last row. ROT_FIX denotes measurements comprising anticlockwise and clockwise rotation of the calibration unit in the cabin and fixed position of about $1 \mathrm{~min}$ before anticlockwise and after clockwise rotation. ROT denotes measurements only during anticlockwise and clockwise rotation

\begin{tabular}{|lllllll|}
\hline & \multicolumn{2}{c}{ April, 10 } & \multicolumn{2}{c}{ June, 19 } & \multicolumn{2}{c|}{ November, 23 } \\
\cline { 2 - 7 } & NUVB & BUVA & NUVB & BUVA & NUVB & BUVA \\
\hline \begin{tabular}{l} 
Stomach: \\
\hline ROT_FIX
\end{tabular} & 1.02 & 1.14 & 0.96 & 1.13 & 0.96 & 1.05 \\
\hline ROT & 0.97 & 1.12 & 0.94 & 1.13 & 0.89 & 1.05 \\
\hline \begin{tabular}{l} 
Chest: \\
\hline ROT_FIX
\end{tabular} & 0.95 & 1.13 & 0.91 & 1.13 & 0.90 & 1.07 \\
\hline ROT & 0.89 & 1.15 & 0.86 & 1.14 & 0.82 & 1.07 \\
\hline Face: & 0.85 & 1.03 & 0.82 & 1.06 & 0.81 & 0.97 \\
\hline ROT_FIX & 0.79 & 1.04 & 0.77 & 1.06 & 0.74 & 0.97 \\
\hline ROT & 945 & 114 & 1143 & 141 & 1485 & 175 \\
\hline Work time [h] & & & & & & \\
\hline
\end{tabular}

- part of a full series including only measurements in periods with CCS rotations (stage 2 and 4 of Mode A).

Doses produced by the narrow band UVB (TL-01) and the broad band UVA (TL-09) tubes were compared in three sessions in 2010 (April 10, June 19, and November 23).

The nominal and observed doses were in good agreement. The ratio (nominal dose divided by the CCS measured dose) was within the range 0.82-1.15 at the level of the stomach and chest (Tables I and II), i.e., for a significant part of the patient's body. The nominal doses assigned in the broad band UVA therapy were only slightly larger than those measured by the CCS, whereas the nominal doses were slightly smaller than the measured ones for the narrow band UVB tubes.

The ratio between the nominal and measured doses in LPC usually decreased when comparing the ratio for doses measured only during the rotating phase of measurements (stage 2 and stage 4)

Table II. Ratio between nominal and measured doses in Poznan cabin (MEDISON 2800-44-PC_AB) during the comparison campaign in April, 2010 for the narrow band UVB TL-01 tube. ROT_FIX and ROT abbreviations are the same as in Table I. FIX denotes the fixed position of the calibration unit in front of the cabin wall

\begin{tabular}{|lccc|}
\hline & ROT_FIX & ROT & FIX \\
\hline Stomach & 0.88 & 0.83 & 0.94 \\
\hline Chest & 0.87 & 0.82 & 0.90 \\
\hline Face & 0.76 & 0.75 & 0.82 \\
\hline
\end{tabular}

with the ratio obtained for the whole Mode A (Table I). More irradiation was received by the CCS in a fixed position. During the rotations, lower irradiation was recorded from the corner zones of the cabin $\left(45^{\circ}, 135^{\circ}, 225^{\circ}\right.$, and $315^{\circ}$, see Figure 2$)$. The difference between the ratios for the rotating phase and the whole Mode A is smaller for the broad band UVA lamp, because TL-09 tubes were spread more evenly in the cabin. The measured UV doses decreased with the height, especially between the chest and face level, and the change was about $10 \%$.

The same pattern of the UV variability inside the cabin was found for PPC in the tests carried out in April 2010, but the ratio between the nominal and measured doses was about $10 \%$ lower (Table II) than that measured during the examination of LPC. The measured doses were closer to the nominal ones, especially at the stomach level, if the CCS was fixed in front of $45^{\circ}-0^{\circ}-315^{\circ}$ cabin wall as it is in Figure 2.

After approx. $500 \mathrm{~h}$ of the narrow band UVB phototherapy and approx. $65 \mathrm{~h}$ of the broad band UVA phototherapy in LPC, there were small changes in the tube efficiency (within 10\% accuracy of the irradiance measurements). Figures 6 and 7 show the time series of the measured irradiance in April, June, and November calibration sessions, for the narrow band UVB and broad band UVA tubes, respectively. Figure 6 A illustrates a slight deterioration ( $10 \%)$ of TL-01 tube efficiency in the 5-month period. The results shown in Figures $6 \mathrm{~B}$ and Figure 7 were obtained during the clockwise (step 4 of Mode A) rotation of the CCS at the height corresponding to the chest level. Figures $6 \mathrm{~B}$ 
A

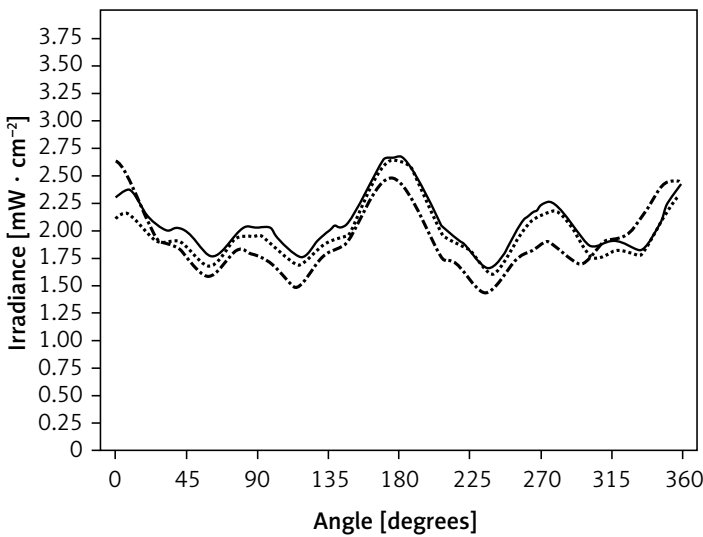

B

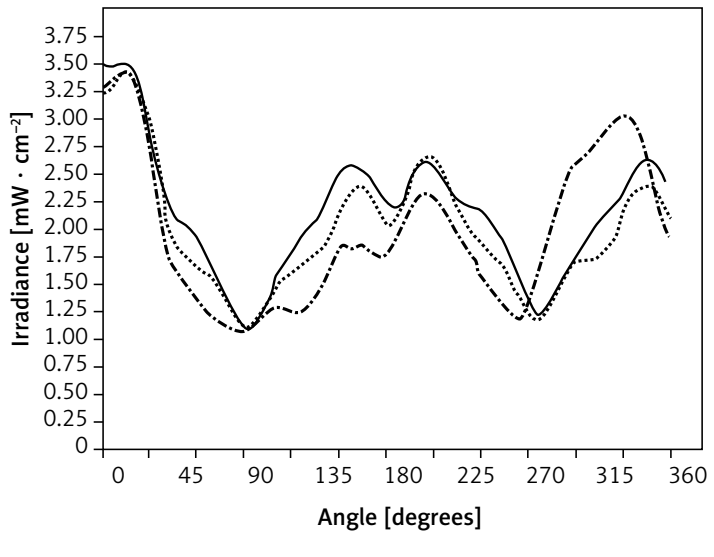

--23.11.2010 - 10.04.2010 -..... 19.06.2010

Figure 6. The irradiance time series measured in the Lodz cabin, with the narrow band UVB TL-01 working tubes, during clockwise rotations of the CCS: YES UVA-1 data (A), NVA-UVB data zoomed on a part of the clockwise rotation (B)

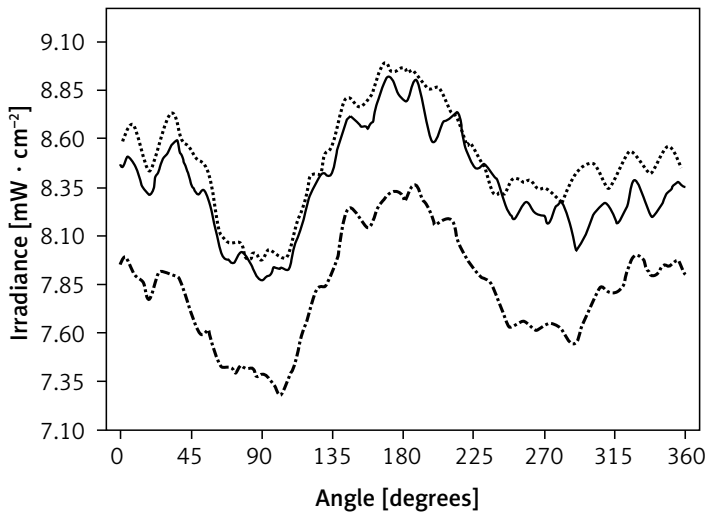

--2 $23.11 .2010 \quad$ - $19.06 .2010 \quad$...... 10.04 .2010

Figure 7. The irradiance time series measured during the clockwise rotations of YES UVA-1 in the calibration sessions of the Lodz cabin with the broad band UVA TL-09 working tubes

Table III. Ratio between nominal and measured doses in Lodz cabin from the comparison session with (or without) a manikin fixed in front of the cabin wall (Figure 4). Three options were examined: without manikin (NO_M), naked manikin ( $M N)$, and dressed manikin (MD). The results are for narrow band UVB TL-01 tubes (NUVB) and broadband UVA TL-09 tubes (BUVA) and two levels of the measuring unit: stomach and chest

\begin{tabular}{|lcccc|}
\hline & \multicolumn{2}{c}{ NUVB } & \multicolumn{2}{c|}{ BUVA } \\
\cline { 2 - 5 } & Stomach & Chest & Stomach & Chest \\
\hline NO_M & 1.23 & 1.18 & 1.19 & 1.21 \\
\hline MN & 1.16 & 1.14 & 1.07 & 1.09 \\
\hline MD & 1.12 & 1.04 & 1.02 & 1.02 \\
\hline
\end{tabular}

shows the irradiance changes during the clockwise rotation between $360^{\circ}$ and $180^{\circ}$ (Figure 2 for the selected cabin sector) by the NVA-UVB meter. The irradiances by TL-09 tubes (Figure 7) decreased only slightly between April 10 and June 19 calibrations, but there was a decrease of about 10\% between June 19 and November 23. The decrease in the measured irradiation may be related to tube aging that may occur abruptly after a period of stable work of the cabin tubes.

Surprisingly, during the November measurements of the cabin irradiation by the narrow band UVB tubes, we found regions (Figure $6 \mathrm{~A}$, around $0^{\circ}-20^{\circ}$, and Figure $6 \mathrm{~B}$, around $315^{\circ}-360^{\circ}$ ) with larger irradiance than was measured in previous intercomparisons. It was explained to us by the nursing staff that two malfunctioning tubes, which were mounted at $45^{\circ}-0^{\circ}-315^{\circ}$ wall, had been replaced with new ones. Signs of tube malfunctioning, i.e., light flickering, had been noticed earlier on (before the November comparison) by a patient.

\section{Attenuation of UV doses by a manikin}

Assessment of the cabin performance requires the irradiance measurements with and without a person in the cabinet to establish a correction factor accounting for the presence of the body, which itself absorbs UV radiation [5]. The body correction factor varies between 0.80 and 0.96 , and it depends on the cabinet design and patient posture $[13,14]$.

Table III shows the comparison between the nominal and measured dose results with a fixed position of the CCS (Mode B) for an empty cabin, an undressed manikin, and a dressed manikin inside the cabin at two heights corresponding to the stomach level (Figure 4 - top) and the chest level (Figure 4 - bottom). The attenuation of the UV dose with the undressed and dressed manikin was more apparent for the UV irradiation by the 
broad band tubes than that for the narrow band ones, i.e.,

- naked manikin: $12 \%$ (both the stomach and the chest level) for the broad band UVA compared to $7 \%$ (stomach), and $4 \%$ (chest) for the narrow band UVB,

- dressed manikin: 17\% (stomach) and 19\% (chest) for the broad band UVA compared to $11 \%$ (stomach) and 14\% (chest) for the narrow band UVB.

The attenuation of the cabin irradiation by the dressed manikin (and the undressed manikin for the broadband UV tubes) was statistically significant, since the uncertainty of the UV irradiance measurements by the CCS was around $10 \%$. The manikin (such as normally used in clothes shops) simulates a rather thin patient, whereas the dressed manikin imitates the presence of a stockier patient in the cabin. The attenuation range found by the CCS in LPC corresponds to the correction factor obtained in earlier studies [14]. Table III shows that the nominal doses set by the nursing staff were about $20 \%$ higher than the ones measured by the CCS for Mode B of LPC testing. The agreement between the doses is better when the cabin is occupied by the manikin. The agreement will be even better for a normal patient. Thus, at the moment we do not recommend using the body correction factor for nominal doses set by nursing staff for the UV exposures in the Lodz cabinet.

\section{Discussion}

The automated system for testing phototherapy cabinets proposed by Curie et al. [8] was used in parallel with manual measurements by a Macam 203 meter to evaluate its performance. Both the automated system and the hand-held UV radiometer were calibrated in the cabin during simultaneous measurements with a high-resolution spectrophotometer. Thus, almost a perfect agreement between doses by the automated system and the radiometer was not surprising. Polish cabinets are not equipped with hand-held personal radiometers, so testing of the cabinet performance could be done comparing the measured UV doses by the proposed system with nominal doses assigned by nursing staff according to medical doctor prescriptions. It is worth mentioning that some authors questioned the effectiveness of the hand-held radiometers for the cabin calibration $[6,10]$.

The CCS is based on the Curie et al. [8] calibration concept of using wide-angle and narrow-angle view sensors rotating inside the cabinet, but different instruments are implemented. The basic instrument of our system, a commercial broadband radiometer routinely used at meteorological stations for UVA monitoring, YES UVA-1, with an almost hemispherical field of view and a small cosine error, is a temperature-stabilized meter (at $45^{\circ} \mathrm{C}$ ). Thus it allows precise measurement of UV radiation in an environment with large temperature variability. The temperature in the centre of the cabin may increase from room temperature at the start to about $40^{\circ} \mathrm{C}$ after $\sim 10$ min' operation. The uncertainty of the cabin UV irradiance measurements by CCS was estimated as 10\%, which is mostly due to the error of estimation of YES UVA-1 instrumental constants $(\sim 6 \%)$ to convert its output in volts to solar irradiance in $\mathrm{mW} \cdot \mathrm{m}^{-2}$ (for more details see supplementary information).

The calibration of our system is done by field comparisons with a routinely operated spectrophotometer (the Brewer spectrophotometer at Central Geophysical Observatory of the Institute of Geophysics, Belsk, Poland) used for continuous measurements of solar UV spectra. Our calibration methodology does not require a set of simultaneous measurements, separately for each lamp category (e.g. narrow band UVB, broadband UVB, broadband UVA), by the system and a high resolution spectrophotometer in a cabinet. The proposed method will have universal applications as it allows one to calibrate any radiometer (with a broad sensitivity band in the UV range) for any kind of UV tube radiation, with known spectral characteristics of its radiation output.

The CCS monitored the performance of phototherapy cabins in Lodz (MEDISUN 2800 PC-AB) and Poznań (MEDISUN-2800-44-PC-AB). The nominal doses $\left(J \cdot \mathrm{cm}^{-2}\right)$ to be received by a patient during the whole body phototherapy, as prescribed by medical doctors, were set differently in the two cabins. In Lodz, the duration of exposure in the cabin was assigned taking into account the value of the prescribed phototherapy dose under the assumption of constant power of the tubes. In Poznan, the appropriate irradiance level and the duration of the phototherapy were adjusted by computer software combined with the builtin meter. Thus, the UV intensity in the cabinet varied throughout phototherapy and the tube aging effect could be compensated by a higher tube voltage. It was found that the UV doses assigned by the nursing staff agreed within $\pm 20 \%$ with the doses measured by the CCS. The UV irradiance at the stomach and chest level was slightly $(\sim 10 \%)$ higher than that measured at the face level. Some parts of the human body therefore could receive significantly different irradiation even at the same level because of the angular changes of UV irradiance inside the cabin. This effect was stronger in Lodz, as the maximum irradiance (in front of the cabin wall) was about $1.0 \mathrm{~mW} \cdot \mathrm{cm}^{-2}$ greater than the minimum irradiance (from the corners of the cabin), whereas the difference between the extremes in Poznan was two times lower due to the 
more evenly distributed UV field owing to more tubes per cabin wall. Thus, for some patients, slow turning around in the cabin during the phototherapy could be a good practice. The examined cabins performed quite well and no corrections of the dosage incident upon a patient were necessary within 5 months of the cabin operation.

The narrow viewing instruments of the CCS are designed to identify changes in the tube efficiency. Originally, its aim was detection of a malfunctioning tube, but during the last calibration in the Lodz cabin (November, 2010) we measured an increase in the cabin irradiation in some sectors after earlier replacement of the malfunctioning tubes with new ones. A patient usually informs the nursing staff of damaged tubes when he sees a flickering light, which is an apparent sign of deteriorating operation of a tube. A slight degradation of tubes' efficiency in the Lodz cabin was detected with hemispherical and narrow viewing instruments. Surprisingly, the cabin aging is more apparent for the broad-band tubes used $\sim 10$ times less frequently than the narrow band (311 nm) tubes. Warming and subsequent cooling of non-operating broad-band lamps by surrounding narrow band tubes may be a reason for the declining tendency. Declining tube efficiency is not a problem in Poznan. Should it occur anyway, the loss in efficiency will be compensated thanks to the computer software that will increase the tube voltage and/or extend the duration of phototherapy.

In conclusion, the measurements of UV radiation inside the cabins by the proposed calibration system provide evidence that real UV doses received by patients during UV phototherapy in Poznan and Lodz agree well (within the range of $\pm 20 \%$ ) with the nominal doses assigned by nursing staff. It does not mean that this will be the case for the same type or other type of cabins using even more sophisticated monitoring of UV irradiance by a built-in dosimeter. Cabinet performance testing always requires independent UV measurements by a well-calibrated device [3]. However, our experiences with the CCS suggest that it will be enough if a calibration of the cabin is performed after $\sim 500 \mathrm{~h}$ of cabin operation. It cannot be assumed that the cabin performance will be consistent over many years of use. UV therapy quality is only guaranteed if the cabinet performance testing is done with a reliable ultraviolet radiometry instrument.

The methodology developed to quantify UV doses in the UVP cabin may be easily extended to measurements of irradiance in the UV-B and UV-A range by any artificial (non-solar) UV radiation source, e.g. UV tubes in solaria. A precondition of starting such measurements is availability of the irradiance spectra of the UV source.

\section{Acknowledgments}

We thank the Tele \& Radio Research Institute, Warsaw, which provided two narrow viewing prototypes used during the cabin calibration sessions. The project was funded by the Ministry of Science and Higher Education under grant no. NN40225336.

\section{References}

1. van Weelden $H$, De La Faille HB, Young E, van der Leun JV. A new development in UVB phototherapy of psoriasis. Br J Dermatol 1988; 119: 11-9.

2. Green C, Diffey BL, Hawk JLM. Ultraviolet radiation in the treatment of skin diseases. Phys Med Biol 1992; 37: 1-20.

3. Taylor DK, Anstey AV, Coleman AJ, et al. Guidelines for dosimetry and calibration in ultraviolet radiation therapy: a report of a British Photodermatology Group workshop. Br J Dermatol 2002; 146: 755-63.

4. Ibbotson S, Bilsland SH, Cox NH, et al. An update and guidance of narrowband ultraviolet $B$ phototherapy: a British Photodermatology group workshop report. $\mathrm{Br}$ J Dermatol 2004; 151: 283-97.

5. Diffey BL, Hart G. Ultraviolet and blue-light phototherapy - principles, sources, dosimetry and safety. Institute of Physics and Engineering in Medicine Report 76, York, 1997.

6. Martin CJ, Curie GD, Pye SD. The importance of radiometer angular response for ultraviolet phototherapy dosimetry. Phys Med Biol 1999; 44: 843-55.

7. Pye SD, Martin CJ. A study of the directional response of ultraviolet radiometers: I. Practical evaluation and implications for ultraviolet measurement standards. Phys Med Biol 2000; 45: 2701-12.

8. Curie GD, Evans AL, Smith D, Martin CJ, McCalman S, Bilsland D. An automated dosimetry system for testing whole-body ultraviolet phototherapy cabinets. Phys Med Biol 2001; 46: 333-46.

9. Moseley H. Scottish UV Dosimetry guidelines "ScUVido". Photodermatol Photoimmunol Photomed 2001; 17: 230-3.

10. Lloyd JJ. Variation in calibration of hand-held UV meters for PUVA and narrowband UVB phototherapy. Br J Dermatol 2004; 150: 1162-6.

11. Henseler T, Hönigsmann H, Wolff K, Christophers E. Oral 8-methoxypsoralen photochemotherapy of psoriasis. The European PUVA study: a cooperative study among 18 European centres. Lancet 1981; 1: 853-7.

12. Lesiak A, Narbutt J, Pawlaczyk M, Sysa-Jędrzejowska A, Krzyścin J. Vitamin D serum level changes in psoriatic patients treated with narrowband ultraviolet B phototherapy are related to the season of the irradiation. Photodermatol Photoimmunol Photomed 2011; 27: 304-10.

13. Langmack KA. An insight into contribution of self-shielding and lamp reflectors to patient exposure in phototherapy units. Phys Med Biol 1988; 43: 207-14.

14. Martin CJ, Clouting H, Aitken A. A study of the correction factor for ultraviolet phototherapy dose measurements made by the indirect metod. Br J Dermatol 2003; 149: 1227-31. 


\section{Appendix 1}

Simultaneous measurements of the solar irradiance by the YES UVA-1 radiometer and the Brewer spectrophotometer (Kipp \& Zonen Inc., Delft, The Netherlands) were carried in 2010 in the Central Geophysical Observatory of the Institute of Geophysics, Polish Academy of Sciences (PAS), at Belsk $\left(52^{\circ} \mathrm{N}, 21^{\circ} \mathrm{E}\right)$. The experiment lasted several days, and its objective was to establish the YES sensitivity to solar irradiance. Additionally, cabin UV spectra were measured in Lodz using a USB2000+ spectrophotometer to calculate an instrumental constant to convert the output of YES UVA-1 (in volts) to irradiance $\left(\mathrm{mW} \cdot \mathrm{cm}^{-2}\right)$ from TL-01 and TL-09 tubes. Figure $1 \mathrm{~B}$ shows normalized spectral sensitivity of the YES UVA-1 meter and the spectra of tube irradiance weighted by the YES sensitivity. The YES UVA-1 radiometer is designed to measure total solar radiation in the UV range, i.e., integral of the solar irradiance spectrum, $I_{\text {SOLAR }}(\lambda)$, over the 290-400 nm range. Its output, $U_{\text {YES }}$, is proportional to the integral of the solar irradiance weighted by the instrument sensitivity to UV,

$\int_{290}^{400} I_{S O L A R}(\lambda) f_{Y E S}(\lambda) d \lambda=\alpha U_{Y E S}$

where $\alpha$ is the instrumental constant, and $f_{Y E S}(\lambda)$ is the spectral instrument sensitivity. The mean value of the instrument spectral response to the surface solar UV radiation, $\left\langle f_{\text {solar }}\right\rangle$, is defined as follows:

$$
\left\langle f_{\text {SOLAR }}\right\rangle=\frac{\int_{290}^{400} I_{\text {SOLAR }}(\lambda) f_{\text {YES }}(\lambda) d \lambda}{\int_{290}^{400} I_{\text {SOLAR }}(\lambda) d \lambda}
$$

Taking into account all ( 6000) solar spectra measured at Belsk by the Brewer spectrophotometer in 2010, it is calculated $\left\langle f_{\text {SOLAR }}\right\rangle=0.589$ $\pm 0.008(2 \sigma)$. Thus, the mean spectral response of the instrument to the solar radiation is almost constant regardless of the seasonal differences in the meteorological conditions over the site.

Regression on the total UV irradiance by the Brewer spectrophotometer, $\int_{290}^{400} I_{\text {SOLAR }}(\lambda) d \lambda$, on output of YES UVA-1, $U_{Y E S}$, during simultaneous measurements by both instruments, allows one to determine the instrumental constant, $\beta$, used in estimations of the total solar UV irradiance from the voltage recorded by YES:

$\int_{290}^{400} I_{\text {SOLAR }}(\lambda) d \lambda=\beta U_{Y E S}$

Figure S1 shows the regression line based on the data collected at Belsk on 12-13 August 2010. Here we found $\beta=24.14 \pm 0.61(2 \sigma) \mathrm{W} \cdot \mathrm{m}^{-2} \cdot \mathrm{V}^{-1}$. The instrumental constant calculated from other experiments was close to this estimate, i.e., 24.07
(July 2, 2010) and 23.65 (2-3 August 2010). After simple mathematical manipulations with (S.1), (S.2), and (S.3) it can be obtained that $\alpha=\beta\left\langle f_{\text {SOLAR }}\right\rangle$. If we select $\beta=24.14$ and $\left\langle f_{\text {SOLAR }}\right\rangle=0.589$ finally we arrive at $\alpha=14.22$.

The uncertainty of this estimate could be $\sim 10 \%$, i.e., $1.3 \%, 2.7 \%$, and $6 \%$ are related to uncertainty of $\left\langle f_{\text {SOLAR }}\right\rangle, \beta$, and the UV spectral irradiance by the Belsk Brewer instrument, respectively. The first and the second percentage values are derived from the range of $2 \sigma$ error of the estimates. The Brewer uncertainty was derived during international intercomparisons of the Brewer instruments in Arosa, Switzerland, in July 2010.

During YES UVA-1 measurements of the UV irradiance in the phototherapy cabin, its specific constant $\alpha$, which provides the individual response of the meter to UV irradiation, will remain the same as that from the solar experiments. The instrument output will reflect changes in the intensity of the cabin irradiation, $I_{C A B I N, K}$, by the fluorescent tubes, i.e.,

$\int_{290}^{400} I_{C A B I N, K}(\lambda) f_{Y E S}(\lambda) d \lambda=\alpha U_{Y E S, K}$

where $K=1, K=2$ denote irradiance by TL-01 and TL-09 tubes, respectively. The mean sensitivity of the YES UVA-1 instrument in the case of the artificial (tube) radiation is defined similarly to that proposed for the solar radiance:

$\left\langle f_{C A B I N, K}\right\rangle=\frac{\int_{290}^{400} I_{C A B I N, K}(\lambda) f_{Y E S}(\lambda) d \lambda}{\int_{290}^{400} I_{C A B I N, K}(\lambda) d \lambda}$

The cabin irradiation spectra due to TL-01 or TL-09 tubes were measured separately using the

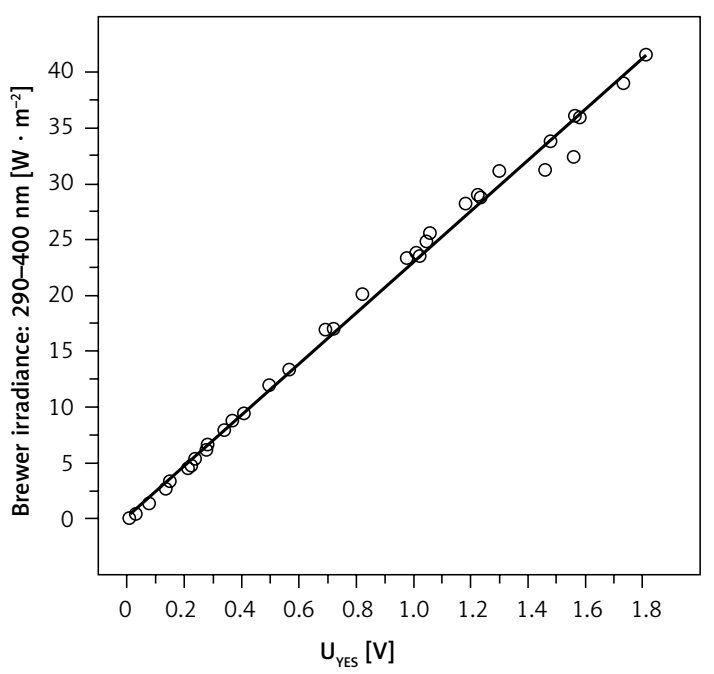

Figure S1. The solar UV irradiance by the Brewer spectrophotometer and output of the YES UVA-1 meter from simultaneous measurements at Belsk, Poland, on August 12/13, 2010. The straight line shows the least-squares regression line 
USB2000+ spectrophotometer. Accordingly (S.5), the mean sensitivity of the YES UVA-1 due to TL01 and TL-09 irradiation is $\left\langle f_{C A B N, 1}\right\rangle=0.735$ and $\left\langle f_{\text {CABIN,2 }}\right\rangle=0.723$.

The UV spectral intensity may differ depending on the measuring site inside the cabin, but the normalized spectral shape (i.e., spectrum divided by its maximum value) is fixed, as it is strictly determined by the photochemical process going in the tube, i.e., gas-discharge triggering fluorescence of the tube luminophore. Thus $\left\langle f_{C A B I N, K}\right\rangle$ is a specific constant pertaining to each type of tube, and its error of estimate is negligible, as the ratio between two integrals in $\mathrm{S} .5$ is sensitive to the lamp spectrum and not affected by the absolute irradiances measured in the cabin.

Finally, after simple algebraic manipulation we obtain:

$\int_{290}^{400} I_{C A B I N, K} d \lambda=\alpha_{K} U_{Y E S, K}, \alpha_{K}=\alpha\left\langle f_{C A B I N, K}\right\rangle^{-1}$

that provides the cabin instrumental constants, $\alpha_{1}=19.35 \mathrm{~W} / \mathrm{m}^{2} / \mathrm{V}$, and $\alpha_{2}=19.67 \mathrm{~W} \cdot \mathrm{m}^{-2} \cdot \mathrm{V}^{-1}$ to be multiplied by measured voltages to obtain the UV irradiance (in $\mathrm{W} \cdot \mathrm{m}^{-2}$ ) for TL-01 and TL-09 tubes, respectively. The uncertainty of $\alpha_{K}$ is calculated as $\sim 10 \%$, which comes from the previous estimation of $\alpha$.

The output of the NVA meters, $U_{N V A, K}$, is in volts. To convert it to the irradiance (expressed in $\mathrm{W}$. $\mathrm{m}^{-2}$ ), the instrumental constants, $\gamma_{K}$, are calculated assuming that the doses recorded by YES and NVA are equal for simultaneous measurements during the rotation of both meters in the cabin:

$\int_{0^{\circ}}^{360^{\circ}} \alpha_{K} U_{Y E S, K} d \varphi=\int_{0^{\circ}}^{360^{\circ}} \gamma_{K} U_{N V A, K} d \varphi$

Finally we obtain the UV irradiance measured by NVA meters as $\gamma_{K} U_{N V A, K}$ where:

$\gamma_{K}=\alpha_{K} \int_{0^{\circ}}^{\int_{0^{\circ}}^{360^{\circ}} U_{Y E S, K}} U_{N, K} d \varphi$

The NVA meter provides the irradiance coming from a small sector of the cabin, and it will be used to identify small-scale spatial variability of UV radiance in the cabin. The absolute value of the irradiance by YES UVA-1 is of special importance, as the radiometer measures radiation coming from the hemispherical area, and its viewing sector is similar to that affecting a patient's body. 